\title{
ОЦЕНКА ЭФФЕКТИВНОСТИ ИСПОЛЬЗОВАНИЯ РЕСУРСНОГО ПОТЕНЦИАЛА ПРЕДПРИЯТИЙ ОБЩЕСТВЕННОГО ПИТАНИЯ НА ОСНОВЕ ДИНАМИЧЕСКОГО ПОДХОДА
}

\author{
(C) 2021 Ломоносова Елена Владимировна \\ старший преподаватель, кафедра бухгалтерского учета, анализа и аудита, \\ Школа экономики и менеджмента \\ Дальневосточный федеральный университет, Россия, Владивосток \\ E-mail lomonosova.ev@dvfu.ru
}

(c) 2021 Олейник Елена Борисовна

доктор экономических наук, кафедра Бизнес-информатики и экономико-математических методов,

Школа экономики и менеджмента

Дальневосточный федеральный университет, Россия, Владивосток

E-mail: oleinik.elena@gmail.com

В статье обоснована необходимость измерения эффекта взаимосвязи процессов использования ресурсного потенциала, представлен способ его расчета. Предложен динамический критерий эффективного использования ресурсного потенциала с учетом особенностей деятельности сферы услуг общественного питания. Проведен анализ эффективности использования ресурсного потенциала предприятия на основе динамического подхода.

Ключевые слова: ресурсный потенциал, эффективность использования ресурсов, эффект взаимосвязи процессов, динамический критерий.

Анализ развития деятельности сферы услуг общественного питания показал, что начиная с 2014 года, наметилась негативная тенденция замедления темпов развития этой сферы услуг, что во многом обусловлено введением разного рода санкций и контрсанкций, в том числе эмбарго на ввоз продуктов питания. Негативная тенденция обострила борьбу за клиента и определила необходимость более глубокого изучения внутренних факторов, основным из которых является эффективное использование ресурсного потенциала предприятий общественного питания.
В результате изучения традиционных инструментов решения задачи повышения эффективности был выявлен их существенный недостаток, связанный с тем, что общепринятые методы не учитывают взаимосвязь экстенсивных и интенсивных факторов и ее влияние на изменение объема производства и реализации продукции и, как следствие, на эффективность использования ресурсного потенциала (рисунок 1).

Для устранения выявленного недостатка, в процессе исследования был детально изучен относительный прирост объема производства

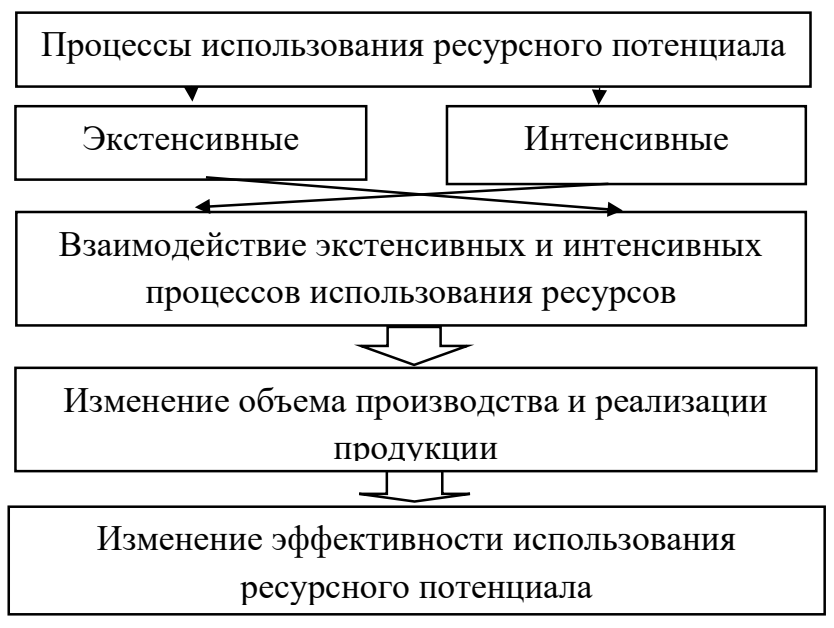

Рисунок 1. Взаимодействие экстенсивных и интенсивных процессов использования ресурсного потенциала и их влияние на изменение эффективности Источник: составлено автором 
и реализации продукции, обусловленный экстенсивным и интенсивным использованием ресурсного потенциала предприятия [3, 4]. В результате выявлено, что относительный прирост объема производства и реализации продукции состоит из трех эффектов, которым были даны названия и сформулированы определения, рисунок 2.

Эффект взаимосвязи процессов на рисунке 2 в традиционных методах не учитывается и не измеряется, то есть его влияние на изменение объема производства и реализации продукции не оценивается. В итоге, положительное значение данного эффекта, увеличивающего объем производства и реализации продукции, достигается случайным образом. В ходе проведенного исследования было выявлено единственное условие, при котором достигается положительное значение эффекта взаимосвязи процессов $[3,4]$. Исходя из этого условия был разработан динамический критерий более эффективного использования ресурсного потенциала, который представляет собой соотношение коэффициентов роста объема производства, интенсивного и экстенсивного процессов использования ресурсного потенциала:

$$
\mathrm{K}_{\mathrm{V}}>\mathrm{K}_{\mathrm{PO}}>\mathrm{K}_{3 \mathrm{P}}>1
$$

где $\mathrm{K}_{\mathrm{V}}$ - коэффициент роста объема производства и реализации продукции; КРо - средний коэффициент роста ресурсоотдачи; К средний коэффициент роста затрат ресурсов.

Исследование показало, что при соотношении коэффициентов роста показателей (1) достигается положительное значение эффекта взаимосвязи процессов, что увеличивает объем производства и реализации продукции и, как следствие, эффективность использования ресурсов.

Учитывая особенности деятельности предприятий общественного питания, в динамический критерий (1) был встроен коэффициент роста показателя фудкост. Показатель фудкост характеризует долю сырьевых затрат в цене блюда [5]. Критерием эффективного использования сырьевых затрат служит снижение показателя фудкост в динамике, что можно представить следующим соотношением:

$$
1>\mathrm{K}_{\phi}
$$

где $\mathrm{K}_{\phi}-$ коэффициент роста показателя фудкост.

Встраивая коэффициент роста показателя фудкост (2) в соотношение коэффициентов роста (1), получим динамический критерий эффективного использования ресурсного потенциала предприятий сферы услуг общественного питания (3):

$$
\mathrm{K}_{\mathrm{V}}>\mathrm{K}_{\mathrm{PO}}>\mathrm{K}_{3 \mathrm{P}}>1>\mathrm{K}_{\phi},
$$

Предложенный динамический критерий (3) позволяет иначе взглянуть на оценку эффективности использования ресурсного потенциала предприятий общественного питания. Одной из особенностей предлагаемого соотношения коэффициентов роста показателей (3), является форма его представления в виде динамического

\begin{tabular}{|c|c|c|}
\hline 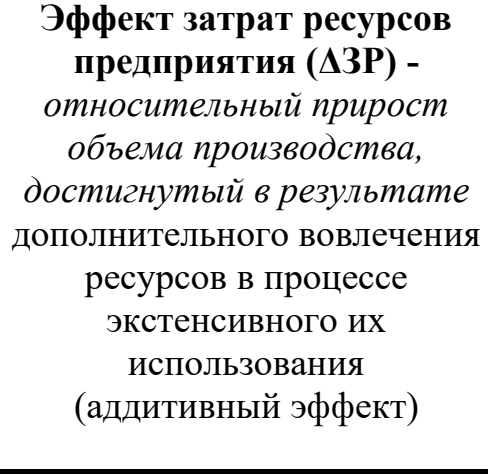 & $\begin{array}{c}\text { Эффект ресурсоотдачи } \\
\text { (АРО) } \\
\text { - относительный прирост } \\
\text { объема производства, } \\
\text { достигнутый в результате } \\
\text { Экономии ресурсов в } \\
\text { процессе интенсивного их } \\
\text { использования } \\
\text { (сверхаддитивный } \\
\text { эффект) }\end{array}$ & $\begin{array}{c}\text { Эффект взаимосвязи } \\
\text { процессов }(\Delta \mathbf{3 P} \times \Delta \mathbf{P O})- \\
\text { относительный прирост } \\
\text { объема производства, } \\
\text { достигнутый в результате } \\
\text { взаимодействия процессов } \\
\text { экстенсивного и } \\
\text { интенсивного использования } \\
\text { ресурсов } \\
\text { (мультипликативный } \\
\text { эффект) }\end{array}$ \\
\hline
\end{tabular}

Относительный прирост объема производства реализации продукции (доли единииы): $\Delta \mathrm{V}=\Delta 3 \mathrm{P}+\Delta \mathrm{PO}+(\Delta 3 \mathrm{P} \times \Delta \mathrm{PO})$

Рисунок 2. Эффекты, реализуемые в процессах экстенсивного и интенсивного использования ресурсного потенциала предприятий Источник: составлено автором 
вектора, принятого в качестве эталонного, идеального. Для выявления степени соответствия фактического соотношения ряда показателей динамическому критерию, необходимо произвести десять сопоставлений. Очевидно, что фактическое соотношение коэффициентов роста показателей не всегда в полной мере будет соответствовать предложенному динамическому критерию (3). Графическая интерпретация близости фактического ряда соотношения показателей с динамическим критерием, представлена на рисунке 3.

Вектор, заданный динамическим критерием, на рисунке (3) обозначен прямой линией $\ni_{\text {рп. }}$ Волнистая линия $Э_{\phi}$ отражает фактическую эффективность использования ресурсного потенциала в каждый момент времени $\mathrm{t}_{\mathrm{n}}$. Чем ближе волнистая линия к прямой линии, тем эффективнее используются ресурсы. Для оценки близости фактического соотношения показателей динамическому критерию, предлагаем рассчитать коэффициенты ранговой корреляции Спирмена и Кендалла.

1. Коэффициент ранговой корреляции по отклонениям Спирмена [2]:

$$
\mathrm{K}_{\text {откл }}=1-\frac{6}{\mathrm{n}\left(\mathrm{n}^{2}-1\right)} \sum_{i=1}^{n}\left(i-R_{i}\right)^{2} \text {, }
$$

где $\mathrm{K}_{\text {откл }}-$ коэффициент ранговой корреляции по отклонениям;

$\mathrm{n}$ - общее количество показателей встроенных в динамический критерий;

i - фактические ранги показателей;
$\mathrm{R}_{\mathrm{i}}$ - эталонные ранги тех же показателей в динамическом критерии.

2. Коэффициент ранговой корреляции по инверсиям Кендалла [2]:

$$
\mathrm{K}_{\text {инв }}=1-\frac{4}{\mathrm{n}(\mathrm{n}-1)} \mathrm{Q} \text {, }
$$

где $\mathrm{K}_{\text {инв }}-$ коэффициент ранговой корреляции по инверсиям;

$\mathrm{n}$ - общее количество показателей встроенных в динамический критерий;

Q - количество неверных соотношений между рангами (количество перестановок).

Коэффициент ранговой корреляции по отклонениям Спирмена следует рассматривать как оценку различия объемной стороны коэффициентов роста показателей в фактическом соотношении и динамическом критерии. Коэффициент ранговой корреляции по инверсиям Кендалла следует рассматривать как оценку различия структуры, т.е. порядка расположения коэффициентов роста показателей в фактическом соотношении и динамическом критерии. Оба коэффициента дают оценку близости ряда фактического соотношения показателей с рядом показателей в динамическом критерии, на интервале от -1 до +1 . Оценка (+1) получается при совпадении фактического ряда с эталонным, а (-1) при их полной разнонаправленности. Интерпретировать значения коэффициентов затруднительно, если не свести к интегральному показателю, предложенному Сыроежкиным И. М. [6].

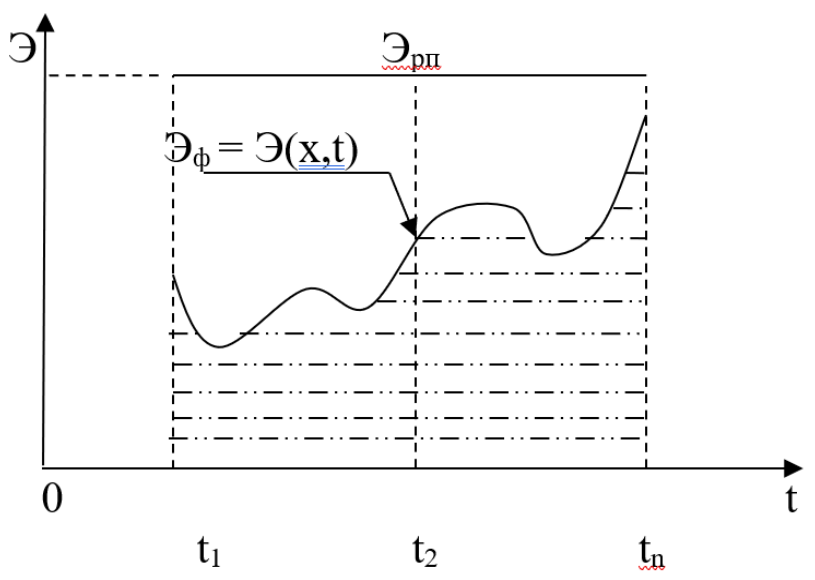

Рисунок 3. Графическая интерпретация близости фактического ряда показателей с динамическим критерием Источник: составлено автором 


$$
\mathrm{R}=\frac{\left(1+\mathrm{K}_{\text {откл }}\right) \times\left(1+\mathrm{K}_{\text {инв }}\right)}{4}
$$

Интегральный показатель R позволяет дать оценку близости фактического соотношения коэффициентов роста, соотношению показателей, встроенных в динамический критерий. Диапазон изменения этого показателя от 0 до +1 . При этом значение показателя (+1) соответствует полному совпадению фактического ряда с эталонным рядом, значение показателя (0) соответствует полному их несовпадению. Эффективное использование ресурсного потенциала отмечается при условии: $\mathrm{R}=100 \%$ (полное соответствие критерию). Значения интегрального показателя, не соответствующие условию эффективного использования, при котором $\mathrm{R}<100 \%$, говорят о необходимости более глубокого и детального рассмотрения и анализа показателей, входящих в динамический критерий, с целью выявления негативных факторов и устранения их влияния.

Продемонстрируем пример оценки эффективности использования ресурсного потенциала ООО «Гавань» на основе расчета интегрального показателя. По данным финансового и управленческого учета рассчитываются коэффициенты роста показателей в динамическом критерии 3, расчеты представлены в таблице 1.

Далее рассчитываются значения коэффициентов Спирмена, Кендалла и интегральный показатель R (таблица 2).

По данным таблицы 2 видно, что эффективно ресурсный потенциал ООО «Гавань» использовался только в 2017 году, данный период принимается за эталон. В 2018 и 2019 годах соответствие критерию составляет $72 \%$, делаем вывод о не эффективном использовании ресурсного потенциала.

Проведем сравнительный анализ оценок эффективности использования ресурсного потенциала с применением общепринятого критерия и предложенного динамического критерия (3). Традиционным критерием эффективного использования ресурсов предприятия служит соотношение коэффициентов роста ресурсоотдачи и затрат ресурсов $\left(\mathrm{K}_{\mathrm{PO}}>\mathrm{K}_{3 \mathrm{P}}\right)$ [1]. Результаты представлены в таблице 3.

По данным таблицы 3 видно, что с примене-

Таблица 1. Коэффициенты роста показателей ООО «Гавань» 2017-2019 гг.

\begin{tabular}{|l|c|c|c|}
\hline \multicolumn{1}{|c|}{ Показатель } & 2017 & 2018 & 2019 \\
\hline Коэффициент роста выручки от продаж & 1,34 & 1,11 & 1,25 \\
\hline Средний коэффициент роста ресурсоотдачи & 1,31 & 1,36 & 2,18 \\
\hline Средний коэффициент роста затрат ресурсов & 1,03 & 0,82 & 0,58 \\
\hline Коэффициент роста фудкост & 0,97 & 0,78 & 0,36 \\
\hline
\end{tabular}

Источник: составлено автором

Таблица 2. Расчетные значения коэффициентов Спирмена, Кендалла и интегрального показателя ООО «Гавань» 2015-2019 гг.

\begin{tabular}{|l|c|c|c|}
\hline \multicolumn{1}{|c|}{ Показатели } & 2017 & 2018 & 2019 \\
\hline Коэффициент Спирмена,\% & 100 & 80 & 80 \\
\hline Коэффициент Кендалла,\% & 100 & 60 & 60 \\
\hline Интегральный показатель R,\% & 100 & 72 & 72 \\
\hline
\end{tabular}

Источник: составлено автором

Таблица 3. Сравнительный анализ оценок эффективности использования ресурсного потенциала 000 «Гавань» 2017-2019 гг.

\begin{tabular}{|c|c|c|c|}
\hline Критерии & 2017 & 2018 & 2019 \\
\hline Предложенный критерий & $\mathrm{R}=100$ & $\mathrm{R}=72$ & $\mathrm{R}=72$ \\
$\mathrm{~K}_{\mathrm{V}}>\mathrm{K}_{\mathrm{PO}}>\mathrm{K}_{3 \mathrm{P}}>1>\mathrm{K}_{\Phi}$ & Эффективно & Не эффективно & Не эффективно \\
$\mathrm{R}=100 \%$ & $1,31>1,025$ & $1,35>0,82$ & $2,18>0,58$ \\
Традиционный критерий & Эффективно & Эффективно & Эффективно \\
\hline
\end{tabular}

Источник: составлено автором 
нием предложенного и традиционного критериев, в 2017 году ресурсный потенциал использовался эффективно. В 2018 и 2019 годах оценки разнятся, с применением предложенного критерия, ресурсный потенциал использовался не эффективно, а с применением традиционного критерия, использовался эффективно.

Применение предложенного динамического критерия, в отличие от традиционного позволяет реализовать положительный эффект взаимосвязи процессов, а также посмотреть на использование ресурсного потенциала предприятий общественного питания шире и выявить негативные факторы, снижающие эффективность использования не только примененных ресурсов, но и потребленных ресурсов.

Для выявления причин несоответствия фактического ряда показателей динамическому критерию, необходимо рассчитать эффекты, входящие в состав относительного прироста объема производства (рисунок 2) и относительный прирост фудкост, результаты представлены в таблице 4.

Судя по данным таблицы 4, в 2018 и 2019 годах причинами несоответствия фактических ря- дов показателей динамическому критерию (3) служат отрицательные эффекты затрат ресурсов, которые обусловили реализацию отрицательных эффектов взаимосвязи процессов. Объем производства продукции в 2018 и 2019 годах уменьшился не только за счет снижения затрат ресурсов на $18 \%$ и $42,5 \%$ соответственно, но и за счет отрицательных эффектов взаимосвязи процессов 6,5\% в 2018 году и 50,2\% в 2019 году.

Значения показателей в таблице 4 служат индикаторами, отражающими слабые звенья в состоянии и использовании ресурсного потенциала предприятия общественного питания, на которые следует обратить внимание и оперативно укрепить их. Для выявления и устранения негативных факторов анализируются структура и динамика затрат ресурсов в эталонном периоде, которые можно перенести на будущий период.

Предложенный метод на основе динамического подхода может применяться в качестве инструмента повышения эффективности использования ресурсного потенциала предприятий общественного питания.

Таблица 4. Составные эффекты относительного прироста объема производства продукции и прирост фудкост 000 «Гавань» 2015-2019 гг.

\begin{tabular}{|c|c|c|c|}
\hline Показатель & 2017 & 2018 & 2019 \\
\hline $\begin{array}{l}\text { 1. Прирост объема производства продукции, } \Delta \mathrm{V} \% \text {, } \\
\text { в том числе: }\end{array}$ & 34,3 & 11,3 & 25,2 \\
\hline а) эффект ресурсоотдачи, $\Delta \mathrm{PO} \%$ & 31,0 & 35,8 & 117,9 \\
\hline б) эффект затрат ресурсов предприятия, $\Delta 3$ Р \% & 2,5 & $-18,0$ & $-42,5$ \\
\hline $\begin{array}{l}\text { в) эффект взаимосвязи процессов, } \\
(\Delta \mathrm{PO} \times \Delta 3 \mathrm{P}) \%\end{array}$ & 0,8 & $-6,5$ & $-50,2$ \\
\hline 2. Прирост фудкост, \% & $-3,1$ & $-22,2$ & $-64,5$ \\
\hline
\end{tabular}

Источник: составлено автором

\section{Библиографический список}

1. Гиляровская Л. Т. Комплексный экономический анализ хозяйственной деятельности: учеб. / Л. Т. Гиляровская [и др.].- М.: ТК «Велби», Изд-во Проспект, 2007.-360 с.

2. Елисеева, Терехов Статистические методы в аудите.- М.: Финансы и статистика, 1998. -176 с.

3. Ломоносова, E. В.Анализ условий реализации мультипликативного эффекта в процессе использования ресурсного потенциала предприятий / Е. В. Ломоносова, В. Г. Сазонов // Известия ДВФУ. Экономика и управление. - 2014.- № 4.- С. 15-28.

4. Ломоносова, Е.В. Условия оптимизации затрат ресурсов предприятий с расчетом эффекта синергии / Е. В. Ломоносова, Е. Б. Олейник // Экономические Науки. - 2018. - № 10 (167). - С. 91-96.

5. Романенко А.В. Методика формирования и оценки показателя «фудскост» на предприятиях общественного питания Вестник ВГУ. Серия Экономика и управление.2019. № 2 с.44-47.

6. Сыроежкин И. М. Совершенствование показателей эффективности и качества.- М.: экономика, 1980.- 192 с. 